\title{
Modalités de prescription des inhibiteurs de la phospodiestérase de type 5
}

\author{
Dominique DELAVIERRE
}

Service d'Urologie - Andrologie, CHR d'Orléans

\section{RESUME}

Les inhibiteurs de la phosphodiestérase de type 5 (PDE 5), sildénafil, tadalafil et vardénafil, sont indiqués dans le traitement de la dysfonction érectile. II s'agit de traitements "à la demande". Le délai recommandé entre la prise du comprimé et la relation sexuelle est de $1 \mathrm{~h}$ avec le sildénafil, $30 \mathrm{mn}$ à $12 \mathrm{~h}$ avec le tadalafil et 25 à $60 \mathrm{mn}$ avec le vardénafil. Une stimulation sexuelle est requise. Les principales contre-indications sont l'association aux dérivés nitrés ou donneurs de monoxyde d'azote (NO) et les troubles cardio-vasculaires sévères pour lesquels une activité sexuelle est déconseillée. Dans certaines situations (association à certains traitements, insuffisance rénale ou hépatique, âge $>65$ ans) les inhibiteurs de PDE 5 ne sont pas recommandés ou peuvent nécessiter une adaptation de la posologie.
Mots-clés : dysfonction érectile, sildénafil, tadalafil, vardénafil
Les inhibiteurs de la phosphodiestérase de type 5 (PDE 5) sont indiqués dans le traitement de la dysfonction érectile définie, depuis le travail de consensus du NIH (National Institute of Health) en 1993, par « l'incapacité d'un homme à obtenir ou maintenir une érection pénienne suffisante pour permettre des rapports sexuels satisfaisants » [6]. IIs ne sont pas indiqués chez l'homme de moins de 18 ans et la femme [7].

\section{Quels sont les produits disponibles ? [1-5]}

Trois inhibiteurs de la PDE 5 sont disponibles sur le marché : le sildénafil (depuis 1998), le tadalafil (depuis 2003) et le vardénafil (depuis 2003 également). Aucun n'est remboursé par la sécurité sociale.

Le sildénafil existe en comprimés bleus non sécables de $25 \mathrm{mg}$ (boîtes de $4 \mathrm{cps}$ ), 50 et $100 \mathrm{mg}$ (boîtes de 4 et 8 cps), le tadalafil en comprimés jaunes non sécables de 10 $\mathrm{mg}$ (boîtes de $4 \mathrm{cps}$ ) et $20 \mathrm{mg}$ (boîtes de 4 et $8 \mathrm{cps}$ ) et le vardénafil en comprimés oranges non sécables de $5 \mathrm{mg}$ (boîtes de $4 \mathrm{cps}$ ), 10 et $20 \mathrm{mg}$ (boîtes de 4 et $8 \mathrm{cps}$ ).

2. Quelles sont les contre-indications des inhibiteurs de la PDE 5 ? [7] (Tableau 1)

Certaines contre-indications sont absolues :

- association aux dérivés nitrés ou donneurs de monoxyde d'azote (NO) ;

- troubles cardio-vasculaires sévères pour lesquels une activité sexuelle est déconseillée (tels qu'angor instable ou insuffisance cardiaque sévère).

\section{Correspondance :}

Dr Dominique DELAVIERRE - Service d'Uro-Andrologie, CHR La Source, BP 6709, 45067 Orléans Cedex 2 - Tel 02.38.51.46.26 - Fax 02.38.51.41.59 - Email delav.uro@wanadoo.fr 
- Association aux dérivés nitrés et donneurs de NO ;

- troubles cardio-vasculaires sévères ;

- infarctus du myocarde et accident vasculaire récents ;

- hypotension artérielle (<90/50 mm HG) ;

- hypertension artérielle non contrôlée (tadalafil) ;

- troubles du rythme non contrôlés (tadalafil);

- insuffisance cardiaque de stade $\geq 2$ selon le NYHA (tadalafil)

- rétinite pigmentaire (sildénafil et vardénafil) ;

- insuffisance hépatique sévère (sildénafil et vardénafil).

Les inhibiteurs de PDE5 sont également contre-indiqués chez certains groupes de patients n'ayant pas été inclus dans les essais cliniques:

- infarctus du myocarde et accident vasculaire récents (les délais recommandés varient, selon l'inhibiteur de la PDE 5 concerné et la pathologie, de 3 à 6 mois) ;

- hypotension artérielle (<90/50 mm HG) ;

- hypertension artérielle non contrôlée (tadalafil) ;

- troubles du rythme non contrôlés (tadalafil) ;

- insuffisance cardiaque de stade $\geq 2$ selon le NYHA (New

York Heart Association) (tadalafil);

- rétinite pigmentaire (sildénafil et vardénafil) ;

- insuffisance hépatique sévère (sildénafil et vardénafil).

D'autres contre-indications sont relatives:

- malformation anatomique du pénis (angulation, sclérose des corps caverneux et maladie de Lapeyronie);

- hémopathies pouvant donner des priapismes (drépanocytose, myélome multiple et leucémie).

\section{Comment prescrire les inhibiteurs de la PDE 5 ? $[1,5]$ (Tableau 2)}

Les inhibiteurs de la PDE 5 sont des traitements ponctuels "à la demande".

La dose initiale recommandée est de $50 \mathrm{mg}$ avec le sildénafil, $10 \mathrm{mg}$ avec le tadalafil et le vardénafil.

Il est préférable de prendre le sildénafil en dehors d'un repas et le vardénafil en dehors d'un repas riche en graisse pour éviter un retard du délai d'action. II n'y a pas de recommandation particulière avec le tadalafil en ce qui concerne les repas.

Le délai recommandé entre la prise du comprimé et la relation sexuelle est de $1 \mathrm{~h}$ avec le sildénafil, $30 \mathrm{mn}$ à $12 \mathrm{~h}$ avec le tadalafil et 25 à $60 \mathrm{mn}$ avec le vardénafil.

Une stimulation sexuelle est requise car les inhibiteurs de la PDE 5 sont des facilitateurs et non des inducteurs de l'érection.
- Posologie initiale recommandée :

- sidénafil : $50 \mathrm{mg}$;

- tadalafil et vardénafil : $10 \mathrm{mg}$.

- Délai recommandé avant la relation sexuelle :

$\begin{array}{ll}\text { - sildénafil : } & 60 \mathrm{mn} ; \\ \text { - tadalafil : } & 30 \mathrm{mn} \text { à } 12 \mathrm{~h} ; \\ \text { - vardénafil : } & 25 \text { à } 60 \mathrm{mn} .\end{array}$

- Stimulation sexuelle requise.

- Pas plus de 1 prise par jour.

II n'est pas recommandé d'aller au-delà d'1 prise par jour et une utilisation quotidienne est déconseillée avec le tadalafil.

\section{Y a t'il des précautions avec certaines associa- tions médicamenteuses ? [7]}

L'association aux dérivés nitrés ou donneurs de NO est formellement contre-indiquée.

L'association aux inhibiteurs des cytochromes P450 3A4 (kétoconazole, itroconazole, erythromycine, clarythromycine, cimétidine, ritonavir, indinavir et saquinavir), selon l'inhibiteur de PDE5 concerné et le traitement associé, est déconseillée ou nécessite d'utiliser la posologie la plus faible. Le jus de pamplemousse est également inhibiteur des cytochromes P3A4 et nécessite les mêmes précautions.

L'association du vardénafil et des alpha-bloquants n'est pas recommandée.

\section{Comment prescrire lors d'insuffisance rénale ou hépatique ? [7] (Tableaux 3 et 4)}

Lors d'insuffisance rénale sévère il convient de débuter le traitement par le sildénafil $25 \mathrm{mg}$ ou le vardénafil $5 \mathrm{mg}$. Quelque soit le degré d'insuffisance rénale, le tadalafil doit être utilisé à la posologie de $10 \mathrm{mg}$.

Lors d'insuffisance hépatique sévère le sildénafil et le vardénafil sont contre-indiqués et lors d'insuffisance hépatique légère ou modérée il convient de débuter le traitement par le sildénafil $25 \mathrm{mg}$ ou le vardénafil $5 \mathrm{mg}$. Quelque soit le degré d'insuffisance hépatique le tadalafil doit être utilisé à la posologie de $10 \mathrm{mg}$.

\section{Comment prescrire chez l'homme de plus de 65 ans ? [7] (Tableau 5)}

Chez l'homme de plus de 65 ans il convient de débuter le traitement par le sildénafil $25 \mathrm{mg}$ ou le vardénafil $5 \mathrm{mg}$. Aucune adaptation n'est requise avec le tadalafil. 


\section{Tableau 3 : Prescription lors d'insuffisance rénale.}

- Sildénafil :

- débuter par 25 mg si insuffisance rénale sévère.

- Vardénafil :

- débuter par $5 \mathrm{mg}$ si insuffisance rénale sévère.

- Tadalafil :

- $10 \mathrm{mg}$ quelque soit le degré d'insuffisance rénale.

Tableau 5 : Prescription chez l'homme de plus de 65 ans.

- Sildénafil :

- débuter par $25 \mathrm{mg}$.

- Vardénafil :

- débuter par $5 \mathrm{mg}$.

- Tadalafil :

- pas d'adaptation.

\section{CONCLUSION}

La prescription d'un inhibiteur de la PDE 5 nécessite certaines précautions et s'effectue en plusieurs étapes (Tableau 6) :

1. Vérification de l'absence de contre-indications ;

2. Choix de la posologie de départ ;

3. Information du patient :

a) Modalités d'utilisation ;

b) Effets secondaires éventuels ;

c) Précautions d'emploi.

4. Remise et explication de l'ordonnance.

Tableau 6 : Les différentes étapes de la prescription.

- Vérification de l'absence de contre-indications.

- Choix de la posologie de départ.

- Information du patient :

- modalités d'utilisation ;

- effets secondaires éventuels ;

- précautions d'emploi.

- Remise et explication de l'ordonnance.
Tableau 4 : Prescription lors d'insuffisance hépatique.

- Sildénafil :

- débuter par $25 \mathrm{mg}$ si insuffisance hépatique légère ou modérée ;

- contre-indiqué si sévère.

- Vardénafil :

- débuter par $5 \mathrm{mg}$ si insuffisance hépatique légère ou modérée,

- contre-indiqué si sévère.

- Tadalafil :

- $10 \mathrm{mg}$ quelque soit le degré de l'insuffisance hépatique.

\section{REFERENCES}

1. BELLA A.J., BROCK G.B. : Tadalafil in the treatment of erectile dysfunction. Curr. Urol. Rep., 2003, $4:$ 472-478.

2. BONDIL P. : Les traitements pharmacologiques. In : Bondil P. ed. La dysfonction érectile. Paris, John Libbey Eurotext, 2003: 137-157.

3. CARSON C.C. $3^{\text {rd }}$ : Sildenafil : a 4-year update in the treatment of 20 million erectile dysfunction patients. Curr. Urol. Rep., 2003, 4 : 488-496.

4. GIULIANO F. : Les nouveaux traitements de la dysfonction érectile après leur mise sur le marché : état des lieux. Communication au forum d'andrologie du 97 ème Congrès Français d'Urologie, 22 novembre 2003, Paris.

5. HELLSTROM W.J. : Vardenafil : a new approach to the treatment of erectile dysfunction. Curr. Urol. Rep., 2003, $4: 479$ 487.

6. NIH : Consensus development panel on impotence. J. Am. Med. Ass., 1993, $270: 83-90$.

7. RCP : (Résumé des Caractèristiques du Produit) VIDAL 2004.

\section{ABSTRACT}

Phosphodiesterase type 5 inhibitors: modalities of prescription

\section{Dominique DELAVIERRE}

Phosphodiesterase type 5 inhibitors, sildenafil, tadalafil and vardenafil, are "on demand" oral treatments for erectile dysfunction. Sildenafil must be taken 1 hour before sexual activity, while tadalafil can be taken $\mathbf{3 0}$ minutes to 12 hours before sexual activity and vardenafil can be taken 25 to 60 minutes before sexual activity. Sexual stimulation is necessary. The main contraindications are the concomitant use of nitric oxide donors or nitrates and serious heart problems (which are a contraindication to sexual intercourse). In some cases (men over the age of 65 , hepatic or renal impairment, association with certain drugs), phosphodiesterase type 5 inhibitors are not recommended or the dosage needs to be adapted. 